\title{
Autophagy is upregulated during colorectal carcinogenesis, and in DNA microsatellite stable carcinomas
}

\author{
PAOLA SENA $^{1 *}$, FRANCESCO MARIANI ${ }^{2 *}$, STEFANO MANCINI $^{2}$, MARTA BENINCASA $^{1}$, \\ GIULIA MAGNANI ${ }^{2}$, MONICA PEDRONI ${ }^{2}$, CARLA PALUMBO $^{1}$ and LUCA RONCUCCI ${ }^{2}$
}

Departments of ${ }^{1}$ Biomedical, Metabolic and Neurosciences, Section of Human Morphology and ${ }^{2}$ Diagnostic and Clinical Medicine, and Public Health, University of Modena and Reggio Emilia, I-41125 Modena, Italy

Received July 7, 2015; Accepted August 19, 2015

DOI: $10.3892 / o r .2015 .4326$

\begin{abstract}
Cancer cells are exposed to a wide range of stress sources, such as nutrient deprivation and hypoxia, as well as cytotoxic chemotherapy and radiotherapy. Certain forms of stress can also promote survival activating the metabolic autophagy pathway in cancer cells. Autophagy is dramatically increased in cancer cells. In these conditions, it is becoming evident that autophagy protects cells, by providing an alternative energy source and by eliminating dysfunctional organelles or proteins. Its role in tumorigenesis is more controversial and both the presence and the absence of autophagy have been implicated. Autophagy is known to be associated with the poor outcome of patients with various types of cancers, and its effectiveness as a prognostic marker in colorectal cancer was demonstrated by several studies. The inhibition of autophagy may be a potential therapeutic target in colorectal cancer. In vitro experiments have shown that the inhibition of autophagy increases 5-FU-induced apoptosis. There are two trials currently investigating the addition of chloroquine to 5-FU-based chemotherapy and bevacizumab. In the present study, we evaluated the expression of LC3B-II in samples of human colorectal microadenomas (i.e., dysplastic aberrant crypt foci) and carcinomas compared to normal mucosa. Furthermore, the expression pattern of LC3B-II was assessed in carcinomas classified as DNA microsatellite stable (MSS) and unstable (MSI). Thus, immunofluorescence techniques coupled with confocal microscopy and immunoblot experiments were performed. The results clearly showed a significant increase in expression of the autophagic key factor in microadenomas and carcinomas with respect to normal mucosa. In
\end{abstract}

Correspondence to: Professor Luca Roncucci, Department of Diagnostic and Clinical Medicine, and Public Health, University of Modena and Reggio Emilia, Via del Pozzo 71, I-41125 Modena, Italy E-mail: luca.roncucci@unimore.it

${ }^{*}$ Contributed equally

Key words: autophagy, colorectal carcinogenesis, microadenoma, aberrant crypt foci, DNA microsatellite instability
MSS carcinomas, the level of LC3B-II expression was higher than that in the MSI carcinomas.

\section{Introduction}

It is an accepted concept of the cancer paradigm that tumorigenesis results from an imbalance between cell proliferation and cell death. It is also becoming clearer that in response to cellular stress, cells may utilize multiple mechanisms that ultimately determine cell death or survival. Cancer cells are exposed to a wide range of stress sources, such as nutrient deprivation and hypoxia, as well as cytotoxic chemotherapy and radiotherapy. Cancer cells utilize several mechanisms to withstand the cell death signals that are generated upon exposure to stress. Cancer cells undergoing certain forms of stress, such as starvation or hypoxia, can also promote survival by modulating their intracellular metabolism (1); for instance activation of the metabolic autophagy pathway. Autophagy or 'cell death occurring with autophagy' is an evolutionarily conserved process aimed at maintaining cellular homeostasis by lysosomal degradation of excessive, damaged and/or aged proteins and organelles. To date, at least three distinct autophagic pathways are known: macroautophagy, microautophagy and chaperone-mediated autophagy. Microautophagy is the sequestration of cytosolic components directly by the lysosomal membrane, whereas chaperone-mediated autophagy involves translocation of targeted proteins directly into the lysosome via a chaperone. Macroautophagy is the most commonly studied form of autophagy in mammalian cells and is characterized by the sequestration of proteins and organelles, such as mitochondria, within an autophagosome (2). Microtubule-associated protein 1 light chain (LC3) is known to be associated with the autophagosomal membrane. The LC3 precursor (pro-LC3) is proteolytically cleaved by Atg4 protease, resulting in the formation of LC3B-I. At the onset of autophagy, LC3B-I is activated and conjugated to an amino group of phosphatidylethanol-amine generating LC3B-II, and then recruited to autophagosomes (3). An increase in LC3 was shown to be directly correlated with the number of autophagosomes and LC3B-II is considered to be the most specific marker of the autophagic process (4).

Autophagy is dramatically increased under stressful (nutrient-deprived) conditions and hypoxia in both normal and 
cancer cells. Under these conditions, it is becoming evident that autophagy protects cells, by providing an alternative energy source and by eliminating dysfunctional organelles or proteins (2). Its role in tumorigenesis is more controversial and both the presence and the absence of autophagy have been implicated (5). Autophagy is known to be associated with the poor outcome of patients with several types of cancers, and its effectiveness as a prognostic marker in colorectal cancer was demonstrated by several studies $(6,7)$.

Colorectal cancer $(\mathrm{CRC})$ is the result of interactions between environmental and genetic factors. Mutations in human DNA mismatch repair (MMR) genes such as $\mathrm{MLH1}, \mathrm{MSH} 2$ and $\mathrm{MSH} 6$ are associated with the development of both hereditary and sporadic CRCs (8). The importance of genetic stabilization by MMR is illustrated by the fact that defects in the human MMR pathway confer a strong predisposition to human hereditary non-polyposis colorectal cancer (HNPCC) and may contribute to the development of $15-20 \%$ of human sporadic CRCs (9). Moreover the DNA MMR system is of clinical importance since mismatch repair-deficient tumor cells are resistant to several classes of commonly used chemotherapy drugs such as the methylating agent temozolomide, the platinum-based drug cisplatin and the antimetabolite 6-thioguanine (6-GT) (10-12).

The inhibition of autophagy has two potential therapeutic targets in CRC. CRC cells have been shown to have functional autophagic machinery that is upregulated during nutrient deprivation, providing an alternative nutrient source and acting as a survival mechanism. Inhibition of this pathway led to marked apoptotic death in CRC cells in vitro (13). Moreover, modulation of autophagy is of interest in overcoming 5-FU resistance. 5-FU-based combination chemotherapy remains the most common drug regimen used in both adjuvant and palliative settings. 5-FU, an anti-metabolite chemotherapy, exerts its cytotoxic effects through both the inhibition of thymidylate synthetase and the incorporation of macromolecules into RNA and DNA, leading to activation of p53 and ultimately apoptosis (14).

In vitro experiments $(15,16)$ have shown that the inhibition of autophagy increases 5-FU-induced apoptosis. There are two trials (NCT01206530, NCT01006369) investigating the addition of chloroquine to 5-FU-based chemotherapy and bevacizumab. As well as assessing efficacy and side-effects, these trials are also aiming to identify surrogate biomarkers for autophagy detection in patient tissue samples and for in vivo molecular imaging (17). This highlights the need for robust biomarkers that reflect functional autophagy and the role of individualized autophagy profiles that can then be used to determine whether to inhibit or induce autophagy.

In the present study, we evaluated the expression of LC3B-II in samples of human colorectal microadenomas and carcinomas compared to normal mucosa. Furthermore, the expression pattern of LC3B-II was assessed in carcinomas classified as DNA microsatellite stable (MSS) and unstable (MSI). Thus, immunofluorescence techniques coupled with confocal microscopy and immunoblot experiments were performed. The results clearly showed a significant increase in the expression of the autophagic key factor in microadenomas and carcinomas with respect to normal mucosa. In MSS carcinomas the level of LC3B-II expression was higher than that in the MSI carcinomas. To our knowledge, this is the first study evaluating the role of autophagy in human colorectal preneoplastic lesions such as microadenomas, and in colorectal carcinomas according to the presence or absence of DNA microsatellite instability; in other words in the presence or deficiency of the DNA mismatch repair pathway.

\section{Materials and methods}

Ethics statement. All patients enrolled in the present study, which underwent colonoscopy or surgical resection for colorectal cancer at the University Hospital of Modena, were asked to provide informed written consent, and the study protocol was specifically approved by the Comitato Etico Provinciale di Modena.

Study population. Forty samples of normal colorectal mucosa (NM) were collected from 20 patients during colonoscopy. All samples were frozen at $-80^{\circ} \mathrm{C}$. All patients had normal colonoscopy results. Thirty microadenomas (MAs) were also identified in 15 patients, and removed after surgery for colorectal cancer on surgical specimens, after staining of the mucosa with a $0.1 \%$ methylene-blue solution in saline, and observation under a dissecting microscope (31). The average multiplicity of the MA examined was 79 (range, 30-120), and all showed low grade dysplasia. All MAs for each patient were frozen at $-80^{\circ} \mathrm{C}$. Finally, 30 samples of colorectal cancer (CRC) were collected from 20 patients operated on for colon or rectal cancer; all samples were frozen at $-80^{\circ} \mathrm{C}$, as described above.

Moreover, we studied histological sections retrieved from pathological archives of 49 carcinomas (CRC). In all 49 samples of carcinoma, the presence of instability at DNA microsatellites was determined (23 were microsatellite stable, MSS, and 26 microsatellite unstable, MSI).

Western blot analysis. One sample frozen at $-80^{\circ} \mathrm{C}$ for each subject was used for western blot analysis. Whole cell lysates were obtained from 20 samples of NM, $15 \mathrm{MA}$, and $15 \mathrm{CRC}$, extracted with hypotonic buffer (50 mM Tris-Cl, $\mathrm{pH} 7.8$, containing $1 \%$ Nonidet P-40, $140 \mathrm{mM} \mathrm{NaCl}, 0.1 \%$ SDS and Na-deoxycholate, $1 \mathrm{mM} \mathrm{Na} \mathrm{VO}_{4}$, and freshly added protease inhibitor cocktail). Lysates were then cleared by centrifugation for $15 \mathrm{~min}$ in a refrigerated centrifuge, max speed, and immediately boiled in SDS sample buffer. An amount of $40 \mathrm{mg}$ of protein extracted from each sample (NM, MA, and CRC) was electrophoresed on SDS-PAGE and transferred to nitrocellulose membranes. The membranes were blocked with $3 \%$ dry milk and 2\% BSA in PBS-T, and incubated with the following antibody, diluted at 1:1,000 overnight at $4^{\circ} \mathrm{C}$ under agitation: [rabbit anti-human MAPLC3 (Santa Cruz Biotechnology)]. After washing, the membranes were incubated with a secondary HPR-conjugated goat anti-mouse IgG antibody $(1: 5,000)$ for $30 \mathrm{~min}$ at room temperature. Immunoreactive proteins were detected with ECL (Amersham). Anti-mouse$\beta$-tubulin (Sigma) was used as loading control. Densitometric analysis was performed using a Kodak Image Station 440cf system (Kodak, Rochester, NY, USA), and semi-quantitative analysis was performed with NIH Image $\mathbf{J}$ software. For each sample and marker, the band intensities were normalized to $\beta$-tubulin, and the results are expressed as the normalized treatment to the control ratio. 
Microsatellite instability. The DNA microsatellite status of all tumors was evaluated using four fluorescent-labeled mononucleotide markers, BAT25, BAT26, NR24 and CAT25. These quasi-monomorphic markers were selected after in-depth review of the literature for their very high sensitivity and specificity in identifying mismatch repair-deficient tumors (18-20). Using this mononucleotide marker panel, a tumor was defined as MSI-positive when showing instability with at least three markers.

DNA from the tumor tissues was amplified in a $10-\mu 1$ volume containing 30-50 ng of DNA, 0.15 pmol of dye-labeled forward and unlabeled reverse primers, $2 \mathrm{mM}$ concentration of each deoxynucleotide triphosphate, $1.5 \mathrm{mM} \mathrm{MgCl}_{2}, 50 \mathrm{mM}$ $\mathrm{KCl}, 10 \mathrm{mM}$ Tris ( $\mathrm{pH} \mathrm{8.3)} \mathrm{and} 0.6$ units of Taq polymerase. Thermocycling conditions were: $94^{\circ} \mathrm{C}$ for $4 \mathrm{~min}$, followed by 11 touchdown cycles, each with a denaturing step at $94^{\circ} \mathrm{C}$ for $30 \mathrm{sec}$, an extension step at $72^{\circ} \mathrm{C}$ for $15 \mathrm{sec}$ and a $75 \mathrm{sec}$ annealing step that decreased $1^{\circ} \mathrm{C} /$ cycle (beginning at $65^{\circ} \mathrm{C}$ in the first cycle and decreasing to $55^{\circ} \mathrm{C}$ in the 11 th cycle). The 11th cycle was then repeated 26 times for a total of 37 cycles of PCR; finally an extension step of $4 \mathrm{~min}$ at $72^{\circ} \mathrm{C}$ followed by storage at $4^{\circ} \mathrm{C}$. PCR products were prepared for analysis by pooling $2 \mu \mathrm{l}$ of dye-D2 reaction, $1 \mu \mathrm{l}$ of dye-D3 and $0.5 \mu \mathrm{l}$ of dye-D4 reaction; $40 \mu \mathrm{l}$ of deionized formamide and $0.5 \mu \mathrm{l}$ of CEQ DNA size standard-400 were added to $0.7 \mu 1$ of the each mixture. All samples were run on a CEQ 8000 sequencer and analyzed using a CEQ 8000 Fragment Analysis system (Beckman Coulter) (21).

Evaluation of immunofluorescence by confocal microscopy. One sample frozen at $-80^{\circ} \mathrm{C}$ for each subject was used for immunofluorescence analysis to evaluate the expression of LC3B-II, vimentin, myeloperoxidase, CD-20 and CD-3 proteins. Twenty samples of NM, 15 MAs and 15 CRCs were fixed in 4\% paraformaldehyde in PBS, cryoprotected in 15\% sucrose in PBS, and frozen in isopentane cooled in liquid nitrogen. Horizontal cryosections of the samples were cut (10 $\mu \mathrm{m}$ thick), and hematoxylin and eosin (H\&E) staining was performed on sections for control tissue integrity and histology. Moreover, the 49 samples of MSS and MSI carcinomas, embedded in paraffin, were used for immunofluorescence analysis as described above. Before immunohistochemistry, routine histology of all tissue samples was carried out after H\&E staining of the sections. Slides were dried overnight at $37^{\circ} \mathrm{C}$, dewaxed in two changes of fresh xylene, and rehydrated in a descending alcohol series. Antigen retrieval involved treatment with a protease (Pronase 1:20; DakoCytomation) for $7 \mathrm{~min}$ at $37^{\circ} \mathrm{C}$. After treatment with $3 \%$ BSA in PBS for $30 \mathrm{~min}$ at room temperature, the cryostatic and paraffin sections were incubated with the primary antibodies: rabbit anti-human MAPLC3 (Santa Cruz Biotechnology); mouse anti-human CD-3, mouse anti-human CD-20, mouse anti-vimentin and mouse anti-myeloperoxidase (Dako), diluted 1:25 in PBS containing 3\% BSA for $1 \mathrm{~h}$ at room temperature. After washing in PBS, the samples were incubated for $1 \mathrm{~h}$ at room temperature with the secondary antibodies diluted 1:20 in PBS containing 3\% BSA [sheep anti-mouse FITCconjugated, goat anti-rabbit TRITC-conjugated (Sigma)]. After washing in PBS and in $\mathrm{H}_{2} \mathrm{O}$, the samples were counterstained with $1 \mathrm{mg} / \mathrm{ml}$ DAPI in $\mathrm{H}_{2} \mathrm{O}$ and then mounted with anti-fading medium (0.21 M DABCO and 90\% glycerol in $0.02 \mathrm{M}$ Tris,
$\mathrm{pH}$ 8.0). Negative control samples were not incubated with the primary antibody. The confocal imaging was performed on a Leica TCS SP2 AOBS confocal laser scanning microscope. Excitation and detection of the samples were carried out in sequential mode to avoid overlapping of signals. Sections were scanned with laser intensity, confocal aperture, gain and black level setting kept constant for all samples. Optical sections were obtained at increments of $0.3 \mathrm{~mm}$ in the z-axis and were digitized with a scanning mode format of $512 \times 512$ or 1,024x1,024 pixels and 256 grey levels. The confocal serial sections were processed with the Leica LCS software to obtain three-dimensional projections. Image rendering was performed by Adobe Photoshop software. The original green fluorescent confocal images were converted to grey-scale and median filtering was performed. An intensity value ranging from 0 (black) to 255 (white) was assigned to each pixel. Background fluorescence was subtracted and immunofluorescence intensity (IF) was calculated as the average for each selected area. The IF of the selected areas, linearly correlated with the number of pixels, was quantitatively analyzed using the standard imaging analysis software of an NIS-Elements system. Each sample was assigned a code number and the score, referred to as immunofluorescence intensity score (IFIS), was determined by an observer who was blinded to the tissue groups during the analysis (22).

Statistical analysis. All quantitative data for NM, MA, and $\mathrm{CRC}$ are reported as mean $\pm \mathrm{SD}$. The average expression difference in the various groups of colorectal lesions, was tested for statistical significance using Kruskal-Wallis analysis, followed by Student-Newman-Keuls test. A value of $\mathrm{P}<0.05$ was chosen to indicate a statistically significant difference.

\section{Results}

Patterns of LC3B-II autophagic activity in the adenoma-carcinoma sequence. In order to evaluate the expression pattern of LC3B-II protein in the samples of normal colorectal mucosa (NM), microadenoma (MA) and colorectal cancer (CRC), immunofluorescence experiments coupled with confocal analysis were performed. Moreover, this technique allowed us to define the accurate distribution pattern of LC3B-II protein in very thick samples, and its immunostaining quantification.

Cells expressing LC3B-II protein showed two distinct autophagic patterns: a diffuse finely and granular reactivity dispersed in the cytoplasm, or a rounded densely stained material, probably enclosed within a cytoplasmic vacuole that accumulated prevalently around the nucleus.

The diffuse granular pattern, often similar to small clumps, was noted in many cancerous epithelial cells of all sections studied, although to a different extent and staining intensity (Fig. 1D).

The dense rounded autophagic vacuoles were well recognizable in stromal cancer cells; such structures varied in size and density, but usually formed coarse, rather than fine, granules (Fig. 1H).

The staining patterns of LC3B-II protein that varied from a diffuse mode to a granular one in the cytoplasm of stromal cells, appeared consistent and unchanged in the same samples, without appreciable variations in intensity and localization 

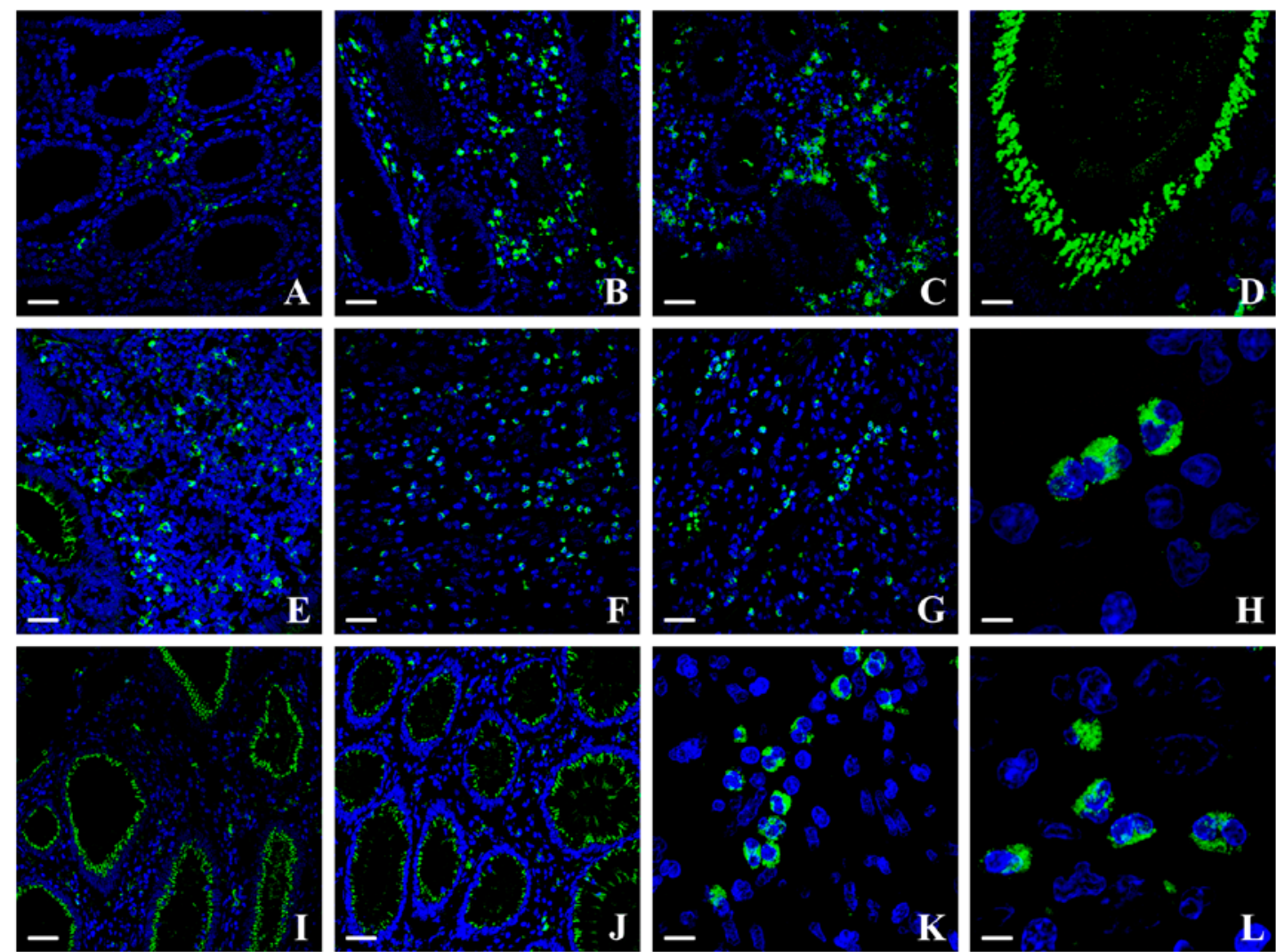

-
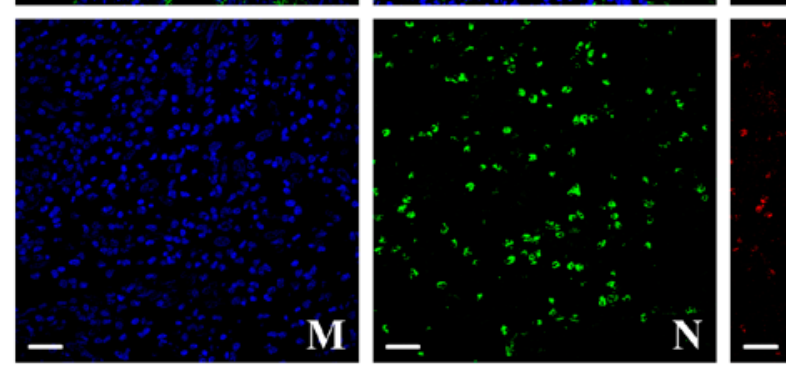

$\mathbf{K}$
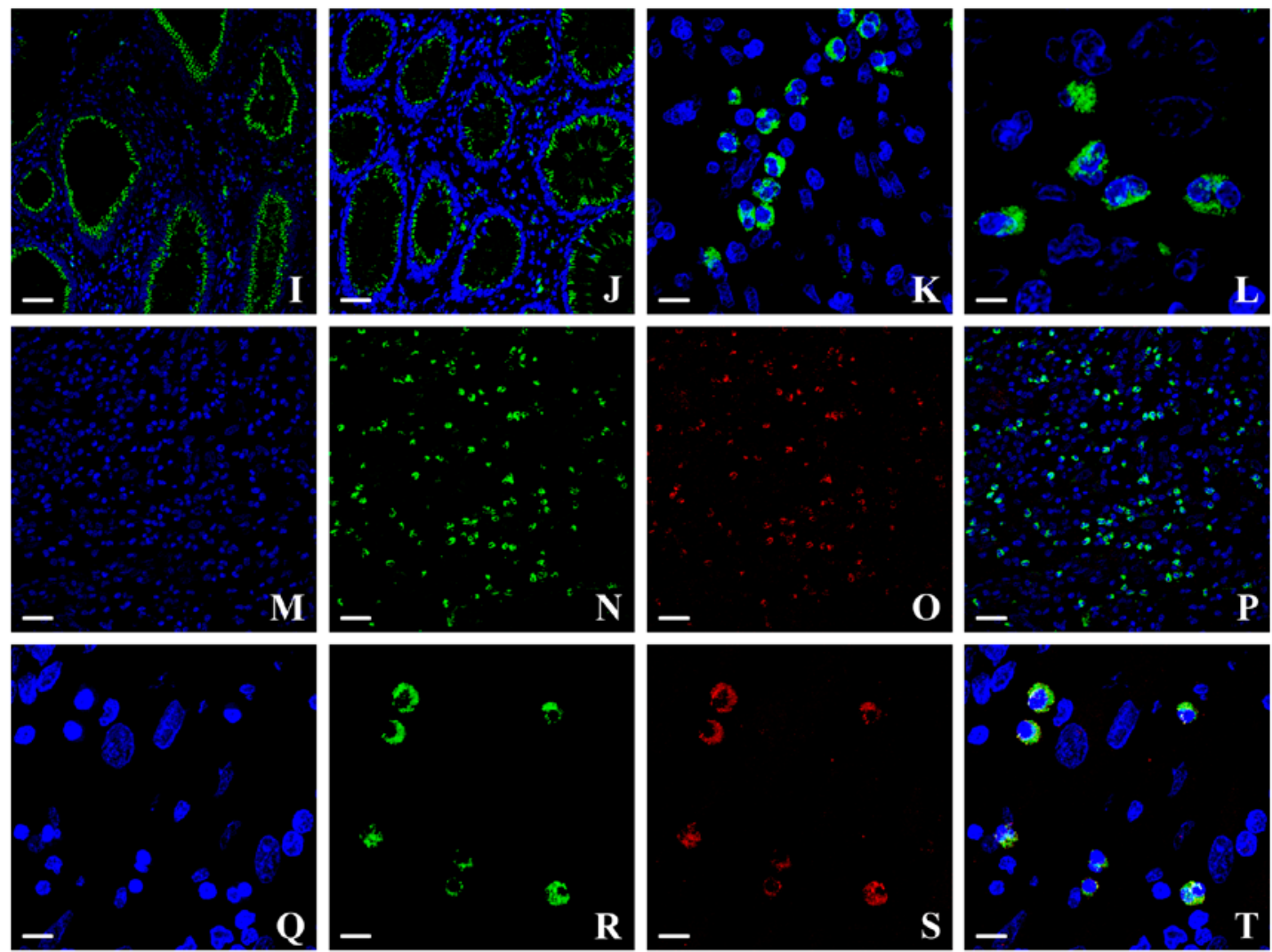

$\mathbf{O}$
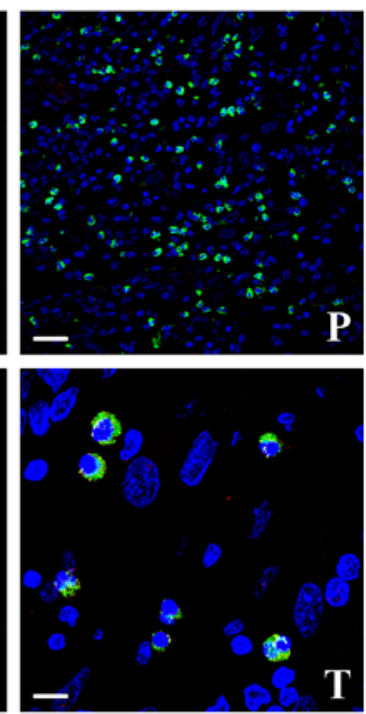

Figure 1. (A-L) Confocal analysis of colonic mucosa cryosections labeled by DAPI (blue) and anti-LC3B-II (green). (A) Normal colorectal mucosa: LC3B-II protein is localized mainly in stromal cells at the cytoplasmic level; the amount of immunostaining is low. (B) Microadenoma: the marked increase in LC3B-II staining with respect to normal mucosa is evident; the staining patterns of LC3B-II protein varies from a diffuse mode to a granular one in the cytoplasm of stromal cells. (C) Colorectal cancer: the immunostaining is significantly enhanced with respect to microadenomas in stromal cells. Many cancerous epithelial cells show marked staining with a diffuse granular pattern (D). Positive cells tended to gather at the edge of the neoplastic tissue (E), in the stroma inside the tumor $(\mathrm{F})$, and also among the neoplastic cells at the margins of the lesions $(\mathrm{G})$. Magnification of stromal cancer cells, the dense rounded autophagic vacuoles were well recognizable (H). (I and J) Confocal analysis of DNA microsatellite stable (MSS) and unstable (MSI) carcinomas. In the epithelium the LC3B-II protein was distributed in a diffuse/granular staining pattern throughout the cytoplasm, accumulated predominantly at the apical region of cells towards the lumen of colonic crypts. The pattern is more evident in MSS tumors (I) than in MSI ones (J). (K and L) Magnification of stromal cells, bright tiny or large aggregates (autophagosomes), are well recognizable (K, MSS; L, MSI). (M-P) Samples of MSS carcinoma stained with DAPI (M, blue), LC3B-II (N, green), vimentin $(\mathrm{O}$, red) and merged $(\mathrm{P})$. In the stromal compartment the presence is evident of many labeled cells with LC3B-II and vimentin, whose co-immunostaining (yellow in P) corresponds to 40\%. (Q-T) Samples of MSS carcinoma stained with DAPI (Q, blue), LC3B-II (R, green), CD-20 (S, red) and merged (T). Only $10 \%$ of T lymphocytes/natural killer cells expressed LC3B-II as revealed by co-immunostaining (yellow in T). Scale bars: A, B, C, E, F, G, I, J, M, N, O and $\mathrm{P}, 50 \mu \mathrm{m} ; \mathrm{D}, 25 \mu \mathrm{m} ; \mathrm{H}$ and $\mathrm{L}, 5 \mu \mathrm{m} ; \mathrm{K}, \mathrm{Q}, \mathrm{R}, \mathrm{S}$ and T, $10 \mu \mathrm{m}$.

among the cells. In all NM samples a few scattered areas in the stromal compartment exhibited a moderate LC3B-II-reactivity, although the epithelial cells were not marked (Fig. 1A).
An intense staining was evident in MA, in stromal cells surrounding colonic crypts, but no appreciable LC3B-II staining of epithelial cells was found (Fig. 1B). 
Table I. Immunofluorescence intensity score (mean \pm SD) of LC3B-II protein in samples of normal colorectal mucosa, microadenoma, colorectal cancer.

\begin{tabular}{lccc}
\hline & NM & MA & CRC \\
\hline IFIS & $37 \pm 5.0$ & $74 \pm 4.0$ & $107 \pm 9.0$ \\
\hline
\end{tabular}

$\mathrm{P}<0.05$ between all group pairs. IFIS, immunofluorescence intensity score; NM, normal colorectal mucosa; MA, microadenoma; CRC, colorectal cancer.

LC3B-II-positive cells were observed throughout the resected tissue in all CRC lesions and they were particularly numerous within the central tumor stroma (Fig. 1C). Concerning the stromal compartment, the samples of CRC were characterized by a higher number of LC3B-II-positive cells with respect to the MAs (Fig. 1B and C); in a subset of carcinoma samples, positive cells were not evenly distributed in the tissue, but tended to gather in some areas, usually at the edge of the neoplastic tissue (Fig. 1E), not only in the stroma inside the tumor (Fig. 1F) but also among the neoplastic cells at the margins of the lesions (Fig. 1G).

Moreover, interestingly, a consistent epithelial positivity was observed in CRC: the protein aggregated in small clumps distributed in the cytoplasm at the lateral and basal portions of the epithelial cells (Fig. 1D and E).

The semi-quantitative evaluation of immunostaining intensity reported as immunofluorescence intensity score (IFIS) (Table I) showed that the level of LC3B-II protein in both epithelial and stromal compartments had an increasing trend from NM to CRC, with statistical significance of the different expression among the groups.

LC3B-II expression pattern in DNA microsatellite stable (MSS) and unstable (MSI) carcinomas. Tumor samples from patients registered in the Colorectal Cancer Registry of Modena, evaluated for DNA microsatellite instability, were analyzed with immunofluorescence techniques, and semi-quantitative evaluation of immunofluorescence staining intensity was performed.

Many stromal cells showed an intense LC3B-II immunoreactivity in the cytoplasm; bright tiny or large aggregates, as expected for autophagosomes, as well as a more diffuse cytoplasmic staining were well recognizable (Fig. 1K and L). The positive stromal cells appeared deeply located, inside the tumor, both in stable and unstable tumor samples.

In the epithelium, LC3B-II protein was distributed homogenously in a diffuse/granular staining pattern throughout the cytoplasm, accumulated predominantly at the apical region of cell towards the lumen of colonic crypts (Fig. 1I and J). These features were more evident in MSS tumors (Fig. 1I) than in MSI ones (Fig. 1J), although positive staining was still detectable in the cells of the surface epithelium and upper glands.

Concerning the semi-quantitative analysis of immunofluorescence intensity, there were substantial differences between stable and unstable tumors. Indeed the IFIS of MSS tumors was $110 \pm 11$ (SD), whereas IFIS of MSI tumors was $89 \pm 11$ (SD); the difference was statistically significant (Table II). In the lamina
Table II. Immunofluorescence intensity score $($ mean \pm SD) of LC3B-II protein in samples of DNA microsatellite stable (MSS) and unstable (MSI) colorectal carcinoma.

\begin{tabular}{lcc}
\hline & MSS & MSI \\
\hline IFIS & $110 \pm 11.0$ & $89 \pm 7.0$ \\
\hline
\end{tabular}

$\mathrm{P}<0.05$. IFIS, immunofluorescence intensity score.

propria of both stable and unstable tumor samples, some infiltrating mononuclear cells were strongly positive for LC3B-II in the cytoplasm.

In order to clarify the role of the stromal compartment in the synthesis of LC3B-II, we tried to identify the type of stromal cells mostly involved in this process. For this purpose, double immunofluorescence analyses were performed with the LC3B-II antibody together with several antibodies specific for different stromal cells, i.e., anti-vimentin, specific for fibroblast-like cells, anti-myeloperoxidase, specific for neutrophils, anti-CD-20 and anti-CD-3 which recognize T and B lymphocytes, respectively. Interestingly, the staining profiles observed showed the highest amount of LC3B-II-positive cells, 40\%, in fibroblast-like cells and $10 \% \mathrm{~T}$ lymphocytes/natural killer cells, as revealed by co-immunostaining with LC3B-II and the antibodies specific for these different cell types (Fig. 1M-T). Very few LC3B-II-positive neutrophils were observed in the tumor stroma. No match with LC3B-II and CD-3 was found. Those percentages were almost the same in the two different types of samples, i.e., stable and unstable tumors.

Western blot analysis. To confirm the findings of the morphological evaluation as described above, cell lysates of NM, MA, and $\mathrm{CRC}$, were subjected to western blot analysis. As shown in Fig. 2A, protein bands immunopositive for LC3-I and LC3-II forms were clearly evident in a representative sample of MA and CRC. Samples of NM generally yielded only a faint band for LC3-I and LC3-II. All tumor tissues showed stronger bands for LC3, especially for LC3-II, compared with the MAs. Densitometric analysis and normalization (with equal amounts of protein loading) of the immunoreactivity signals from protein extracts of NM, MA and CRC showed that the level of LC3-I and LC3-II proteins had an increasing trend from NM to CRC, with statistical significance of the different expression levels (Fig. 2B).

\section{Discussion}

To underline the novelty of our study, the following main findings of the present investigation are summarized as follows. i) The staining patterns of LC3B-II protein varied both in morphological and quantitative aspects during the neoplastic progression of human intestinal mucosa. The results clearly outline that there is a striking increase in the intensity and density of staining for LC3B-II protein in the normal mucosa-microadenoma-carcinoma sequence. Immunofluorescence analyses showed that LC3B-II was expressed in normal mucosa, although at low levels, whereas 
A

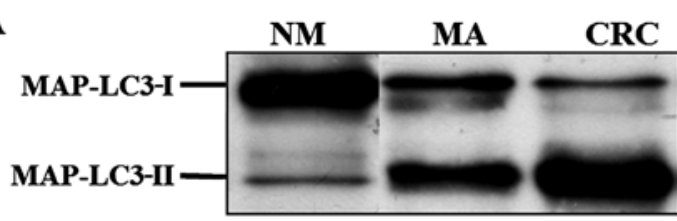

B
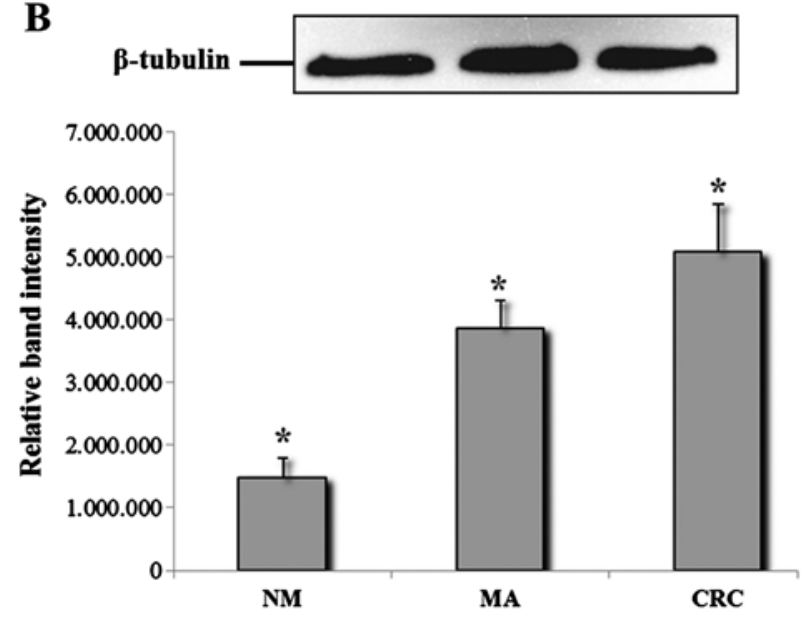

Figure 2. (A) Western blot analysis of normal colorectal mucosa (NM), microadenoma (MA), and colorectal cancer (CRC) samples, using anti-LC3B-II antibody. (B) Mean densitometric data of LC3B-II expression were analyzed using a NIH Image $\mathrm{J}$ software. ${ }^{*} \mathrm{P}<0.05$ between all group pairs of samples. Bar indicates SD.

it was significantly increased in microadenoma and in cancer. ii) The results obtained with immunofluorescence analyses were confirmed by western blotting data, with no difference according to the various techniques used. iii) In DNA microsatellite stable and unstable tumors, a strong positive staining was detectable both in epithelial and stromal compartments, and there were substantial differences between stable and unstable tumors in the semi-quantitative analysis of immunofluorescence. In MSS carcinomas, the level of LC3B-II expression was higher than that in MSI carcinomas, and LC3B-II-positive cells were mostly (40\%) fibroblast-like cells.

The first point to emphasize is that the aim of this study was to deepen the knowledge of the relationship between autophagy and human CRC progression from the early steps of this process. To our knowledge, this is the only study in which the LC3B-II expression pattern in DNA microsatellite stable and unstable CRCs was investigated.

Emerging evidence has shown that tumorigenesis and the progression of human cancers are affected by disturbances in the molecular machinery regulating autophagy $(5,23)$. However, the role of autophagy in cancer development and progression remains controversial and may be regarded as a double-edged sword. Recycling old and damaged cellular material induced by stress and toxic injuries, in addition to providing an alternative energy source, could be seen as assisting cancer cell survival. On the other hand, when autophagy is pushed beyond a crucial 'switch' point, it can lead to cell death which, if harnessed, would be a useful mechanism for eradication of tumor cells. Autophagy is considered a form of programmed cell death (type II) responsible for eliminating damaged and/ or harmful cells such as cancer cells treated with anticancer reagents. Several studies citing the pros and cons of autophagy in the cancer context may be found in the scientific literature. Autophagy can be involved in either the promotion or inhibition of cancer cell survival $(24,25)$. Experimental studies have indicated that in the case of CRC cell lines, that were resistant to nutrient deprivation culture conditions, autophagy may provide an alternative source of energy through degradation of organelles (13). Degraded material would either be coverted into energy or biomass production, and the energy aspect would certainly benefit CRC cells which are under hypoxia when the blood supply is limited in the central portion of the tumor mass. Interestingly, we found that the expression levels of LC3B-II were increased in precancerous lesions (i.e., microadenomas), with respect to normal mucosa. The increment was statistically significant and it was even more evident in the carcinoma samples. These data are in keeping with the results reported in our previous study, in which we demonstrated a gradual increase in hypoxia-inducible factor- $1 \alpha$ $(\mathrm{HIF}-1 \alpha)$ protein expression in microadenomas, adenomas and carcinomas, showing a strong relation to dysplasia (26). HIF- $1 \alpha$ is a key transcription factor that accumulates under hypoxic conditions (27). The importance of the association between hypoxia/HIF-1 $\alpha$ and autophagy has previously been demonstrated $(28,29)$. This relationship seems to indicate that hypoxia, an early event in tumor growth, may trigger autophagy during malignant transformation (30). Moreover, we showed a marked downregulation of apoptosis in human colorectal microadenomas with respect to normal mucosa (31). It is an accepted feature of the cancer paradigm that tumorigenesis results from an imbalance between cell proliferation and cell death. It is also becoming clearer that in response to cellular stress, cells may utilize multiple mechanisms that ultimately determine cell death or survival. Apoptosis and autophagy are regulated by common upstream signals, share common components, and there is also a functional relationship between autophagy and apoptosis (32). Regarding cancer development and treatment, the two processes commonly occur in the same cells; in some cases inhibition of apoptosis causes autophagy (33), while, in other cases, inhibition of autophagy triggers apoptosis (34). Taken together our findings indicate that autophagy can be viewed as a survival pathway to promote cancer development, especially given the fact that the biomarker of autophagy is strongly detectable from the early stage of CRC progression. Thus targeting autophagy may be a useful strategy for cancer treatment. These observation are in keeping with those of Yoshioka et al (35).

Moreover, autophagy is known to be associated with poor outcome in several types of cancers and several studies have also demonstrated its effectiveness as a prognostic marker in CRC $(6,7,36,37)$. Autophagy is reported to be upregulated and associated with drug resistance in various treatment modalities for CRC (15). This process can prevent or promote colorectal carcinogenesis as well as modulate the response to various treatments $(38,39)$. Therapeutic research and clinical trials are currently underway to target both these properties (40). Another interesting finding of our study is the analysis of LC3B-II in samples of DNA microsatellite stable and unstable carcinomas. The DNA mismatch repair system plays a critical role in mutation avoidance and tumor suppression. The impor- 
tance of genetic stabilization by MMR is illustrated by the fact that defects in the human MMR pathway confer a strong predisposition to human hereditary non-polyposis colorectal cancer (HNPCC) and may contribute to the development of $15-20 \%$ of human sporadic CRCs (41). The MSI phenotype is characterized by a very specific clinicopathological profile. Colorectal tumors with MSI are more often localized in the right colon and are associated with high histological grade, mucinous component, tumor-infiltrating lymphocytes, more necrosis and the presence of a Crohn's like host response (42). Thus, it was interesting to see whether these histological findings had an impact on patient outcome. A better prognosis of MSI tumors has been reported in studies describing this genetic instability in CRC $(43,44)$, as in further retrospective studies (45-49). However, many of the studies were of small sample size and included stage I-IV cancers and/or patients treated by adjuvant or palliative chemotherapy. Studies with a larger population restricted to stages II and III and randomized versus no treatment are crucial to demonstrate the prognostic significance of the altered MMR system in resected CRC. However, research recently showed that most (90\%) MSI tumors were part of the low risk group among stage II colon cancer patients classified by the prognostic ColoPrint ${ }^{\circledR}$ gene expression signature in a series of 114 stage II tumors (50). Moreover, MSI remained a strong independent prognostic marker in a multivariate analysis including the other well-known prognostic Oncotype ${ }^{\circledR}$ gene expression signature performed in 1,436 stage II colon cancers from the QUASAR study $(51,52)$. Mechanisms underlying the favorable prognosis of MSI CRC remain widely unknown. The presence of a local antitumor cytotoxic immune response due to the high content of tumor-infiltrating lymphocytes that characterizes these tumors may partially explain their better clinical outcome (53). This immune response also probably explains the higher number of lymph nodes found in resected samples of MSI stages I and II CRC compared to their MSS counterpart (54). This study showed a significant difference in the semi-quantitative analysis of LC3-II protein expression pattern, i.e., the higher intensity of fluorescence in MSS carcinomas with respect to MSI carcinomas in the epithelium of colon cancer cells. Another point of the present study was the identification of the types of stromal cells producing LC3-II proteins in MSS and MSI carcinomas, which has never been previously reported. LC3-II was expressed by stromal fibroblasts in a high percentage and by $\mathrm{T}$ lymphocytes/natural killer cells in smaller amounts. Several studies using mouse models of genetically altered fibroblasts demonstrated a direct involvement of resident fibroblasts in the onset of cancer (55); moreover, both genetic and cell-biology studies indicate that tumor growth is not just determined by malignant cancer cells themselves, but also by the tumor stroma (56). The expression of autophagy-related proteins in the stroma can be explained by the reverse Warburg effect - the interaction between tumor and stromal metabolism in breast cancer. According to this hypothesis, reactive oxygen species from breast cancer cells induce stromal glycolysis, mitochondrial dysfunction, and increased autophagy. Ketone bodies and lactate resulting from stromal cell glycolysis enter the tumor cell, which generates ATP through oxidative phosphorylation. Therefore, according to this theory, the expression of LC3B-II we observed in the stroma may reflect increased stromal autophagic activity.
Thus, the cells interacting with the tumor cells are probably cancer-associated fibroblasts (CAFs) (57-59). CAFs represent the major cellular component of the desmoplastic stroma of solid cancers and their metastases (60).

In clinical practice, two molecular features are currently used in order to select the best therapeutic approach in CRC patients. The first is the DNA microsatellite status and second is the KRAS status. KRAS is mutated in a substantial part of all CRCs, with a particular increased prevalence in MSSs, while when we consider only MSIs this prevalence drops. This is of great interest for our study, since we demonstrated that autophagy is significantly higher in MSSs than in MSIs. Thus, a clinical evaluation of the expression of autophagy in CRC patients could play a role in selecting potential responders or non-responders to autophagy inhibitory drugs. In conclusion, we elucidated the expression pattern of an autophagy-related protein in the adenoma-carcinoma sequence of the human large bowel, investigating the relationship between the autophagic process and the DNA microsatellite status. Our data suggest that autophagy is activated in human CRC cells and may represent a pro-survival mechanism by enhancing the ability of cancer cells to adapt to apoptotic stimuli and hypoxic conditions during cancer progression.

\section{Acknowledgements}

The present study was supported by funds of the Associazione per la Ricerca sui Tumori Intestinali (ARTI), which also provided support to G.M. The authors wish to thank the Centro Interdipartimentale Grandi Strumenti (CIGS) of the University of Modena and Reggio Emilia, for software, instrument availability and assistance.

\section{References}

1. Kondo Y, Kanzawa T, Sawaya R and Kondo S: The role of autophagy in cancer development and response to therapy. Nat Rev Cancer 5: 726-734, 2005.

2. Mizushima N, Ohsumi Y and Yoshimori T: Autophagosome formation in mammalian cells. Cell Struct Funct 27: 421-429, 2002.

3. Kabeya Y, Mizushima N, Ueno T, Yamamoto A, Kirisako T, Noda T, Kominami E, Ohsumi Y and Yoshimori T: LC3, a mammalian homologue of yeast Apg8p, is localized in autophagosome membranes after processing. EMBO J 19: 5720-5728, 2000.

4. Mizushima N, Yoshimori T and Levine B: Methods in mammalian autophagy research. Cell 140: 313-326, 2010.

5. Mathew R, Karantza-Wadsworth V and White E: Role of autophagy in cancer. Nat Rev Cancer 7: 961-967, 2007.

6. Giatromanolaki A, Koukourakis MI, Harris AL, Polychronidis A, Gatter KC and Sivridis E: Prognostic relevance of light chain 3 (LC3A) autophagy patterns in colorectal adenocarcinomas. J Clin Pathol 63: 867-872, 2010.

7. Park JM, Huang S, Wu TT, Foster NR and Sinicrope FA: Prognostic impact of beclin 1, p62/sequestosome 1 and LC3 protein expression in colon carcinomas from patients receiving 5-fluorouracil as adjuvant chemotherapy. Cancer Biol Ther 14: 100-107, 2013.

8. Buermeyer AB, Deschênes SM, Baker SM and Liskay RM: Mammalian DNA mismatch repair. Annu Rev Genet 33: 533-564, 1999.

9. Jacob S and Praz F: DNA mismatch repair defects: Role in colorectal carcinogenesis. Biochimie 84: 27-47, 2002.

10. Modrich P and Lahue R: Mismatch repair in replication fidelity, genetic recombination, and cancer biology. Annu Rev Biochem 65: 101-133, 1996

11. Fink D, Aebi S and Howell SB: The role of DNA mismatch repair in drug resistance. Clin Cancer Res 4: 1-6, 1998. 
12. Stojic L, Brun R and Jiricny J: Mismatch repair and DNA damage signalling. DNA Repair (Amst) 3: 1091-1101, 2004.

13. Sato K, Tsuchihara K, Fujii S, Sugiyama M, Goya T, Atomi Y, Ueno T, Ochiai A and Esumi H: Autophagy is activated in colorectal cancer cells and contributes to the tolerance to nutrient deprivation. Cancer Res 67: 9677-9684, 2007.

14. Longley DB, Harkin DP and Johnston PG: 5-fluorouracil: Mechanisms of action and clinical strategies. Nat Rev Cancer 3: $330-338,2003$

15. Li J, Hou N, Faried A, Tsutsumi S, Takeuchi T and Kuwano H: Inhibition of autophagy by 3-MA enhances the effect of 5-FU-induced apoptosis in colon cancer cells. Ann Surg Oncol 16: 761-771, 2009.

16. Li J, Hou N, Faried A, Tsutsumi S and Kuwano H: Inhibition of autophagy augments 5-fluorouracil chemotherapy in human colon cancer in vitro and in vivo model. Eur J Cancer 46 : 1900-1909, 2010

17. Sui X, Chen R, Wang Z, Huang Z, Kong N, Zhang M, Han W, Lou F, Yang J, Zhang Q, et al: Autophagy and chemotherapy resistance: A promising therapeutic target for cancer treatment. Cell Death Dis 4: e838,2013.

18. Suraweera N, Duval A, Reperant M, Vaury C, Furlan D, Leroy K, Seruca R, Iacopetta B and Hamelin R: Evaluation of tumor microsatellite instability using five quasimonomorphic mononucleotide repeats and pentaplex PCR. Gastroenterology 123 : 1804-1811, 2002

19. Xicola RM, Llor X, Pons E, Castells A, Alenda C, Piñol V, Andreu M, Castellví-Bel S, Payá A, Jover R, et al; Gastrointestinal Oncology Group of the Spanish Gastroenterological Association: Performance of different microsatellite marker panels for detection of mismatch repair-deficient colorectal tumors. J Nat Cancer Inst 99: 244-252, 2007

20. Deschoolmeester V, Baay M, Wuyts W, Van Marck E, Van Damme N, Vermeulen P, Lukaszuk K, Lardon F and Vermorken JB: Detection of microsatellite instability in colorectal cancer using an alternative multiplex assay of quasi-monomorphic mononucleotide markers. J Mol Diagn 10: 154-159, 2008.

21. Pedroni M, Roncari B, Maffei S, Losi L, Scarselli A, Di Gregorio C, Marino M, Roncucci L, Benatti P, Ponti G, et al: A mononucleotide markers panel to identify hMLH1/hMSH2 germline mutations. Dis Markers 23: 179-187, 2007.

22. Mariani F, Sena P, Pedroni M, Benatti P, Manni P, Di Gregorio C, Manenti A, Palumbo C, de Leon MP and Roncucci L: Th inducing POZ-Kruppel Factor (ThPOK) is a key regulator of the immune response since the early steps of colorectal carcinogenesis. PLoS One 8: e54488, 2013

23. Mizushima N, Levine B, Cuervo AM and Klionsky DJ: Autophagy fights disease through cellular self-digestion. Nature 451: $1069-1075,2008$

24. Liang XH, Jackson S, Seaman M, Brown K, Kempkes B, Hibshoosh $\mathrm{H}$ and Levine B: Induction of autophagy and inhibition of tumorigenesis by beclin 1. Nature 402: 672-676, 1999 .

25. Wang J: Beclin 1 bridges autophagy, apoptosis and differentiation. Autophagy 4: 947-948, 2008.

26. Mariani F, Sena P, Marzona L, Riccio M, Fano R, Manni P, Gregorio CD,Pezzi A,Leon MP,Monni S, et al: Cyclooxygenase-2 and hypoxia-inducible factor-lalpha protein expression is related to inflammation, and up-regulated since the early steps of colorectal carcinogenesis. Cancer Lett 279: 221-229, 2009.

27. Beasley NJ, Wykoff CC, Watson PH, Leek R, Turley H, Gatter K, Pastorek J, Cox GJ, Ratcliffe P and Harris AL: Carbonic anhydrase IX, an endogenous hypoxia marker, expression in head and neck squamous cell carcinoma and its relationship to hypoxia, necrosis, and microvessel density. Cancer Res 61: 5262-5267, 2001

28. Mizushima N: Methods for monitoring autophagy. Int J Biochem Cell Biol 36: 2491-2502, 2004

29. Jin S and White E: Role of autophagy in cancer: Management of metabolic stress. Autophagy 3: 28-31, 2007

30. Giatromanolaki A, Koukourakis MI, Koutsopoulos AV Harris AL, Gatter KC and Sivridis E: Autophagy and hypoxia in colonic adenomas related to aggressive features. Colorectal Dis 15: e223-e230, 2013.

31. Sena P, Roncucci L, Marzona L, Mariani F, Maffei S, Manenti A and De Pol A: Altered expression of apoptosis biomarkers in human colorectal microadenomas. Cancer Epidemiol Biomarkers Prev 19: 351-357, 2010.

32. Inbal B, Bialik S, Sabanay I, Shani G and Kimchi A: DAP kinase and DRP-1 mediate membrane blebbing and the formation of autophagic vesicles during programmed cell death. J Cell Biol 157: 455-468, 2002.
33. Degenhardt K, Mathew R, Beaudoin B, Bray K, Anderson D, Chen G, Mukherjee C, Shi Y, Gélinas C, Fan Y, et al: Autophagy promotes tumor cell survival and restricts necrosis, inflammation, and tumorigenesis. Cancer Cell 10: 51-64, 2006.

34. Amaravadi RK, Yu D, Lum JJ, Bui T, Christophorou MA, Evan GI, Thomas-Tikhonenko A and Thompson CB: Autophagy inhibition enhances therapy-induced apoptosis in a Myc-induced model of lymphoma. J Clin Invest 117: 326-336, 2007.

35. Yoshioka A, Miyata H, Doki Y, Yamasaki M, Sohma I, Gotoh K, Takiguchi S, Fujiwara Y, Uchiyama Y and Monden M: LC3, an autophagosome marker, is highly expressed in gastrointestinal cancers. Int J Oncol 33: 461-468, 2008.

36. Guo GF, Jiang WQ, Zhang B, Cai YC, Xu RH, Chen XX, Wang F and Xia LP: Autophagy-related proteins Beclin-1 and LC3 predict cetuximab efficacy in advanced colorectal cancer. World J Gastroenterol 17: 4779-4786, 2011.

37. Miao Y,Zhang Y, Chen Y, Chen L and Wang F: GABARAP is overexpressed in colorectal carcinoma and correlates with shortened patient survival. Hepatogastroenterology 57: 257-261, 2010.

38. White E, Karp C, Strohecker AM, Guo Y and Mathew R: Role of autophagy in suppression of inflammation and cancer. Curr Opin Cell Biol 22: 212-217, 2010

39. Chen N and Karantza V: Autophagy as a therapeutic target in cancer. Cancer Biol Ther 11: 157-168, 2011.

40. Hönscheid P, Datta K and Muders MH: Autophagy: Detection, regulation and its role in cancer and therapy response. Int $\mathrm{J}$ Radiat Biol 90: 628-635, 2014.

41. van Lier MG, Leenen CH, Wagner A, Ramsoekh D, Dubbink HJ, van den Ouweland AM, Westenend PJ, de Graaf EJ, Wolters LM, Vrijland WW, et al; LIMO Study Group: Yield of routine molecular analyses in colorectal cancer patients $\leq 70$ years to detect underlying Lynch syndrome. J Pathol 226: 764-774, 2012.

42. Jass JR, Do KA, Simms LA, Iino H, Wynter C, Pillay SP, Searle J, Radford-Smith G, Young J and Leggett B: Morphology of sporadic colorectal cancer with DNA replication errors. Gut 42: 673-679, 1998

43. Thibodeau SN, Bren G and Schaid D: Microsatellite instability in cancer of the proximal colon. Science 260: 816-819, 1993.

44. Lothe RA, Peltomäki P, Meling GI, Aaltonen LA, NyströmLahti M, Pylkkänen L, Heimdal K, Andersen TI, Møller P, Rognum TO, et al: Genomic instability in colorectal cancer: Relationship to clinicopathological variables and family history. Cancer Res 53: 5849-5852, 1993.

45. Choi SW, Lee KJ, Bae YA, Min KO, Kwon MS, Kim KM and Rhyu MG: Genetic classification of colorectal cancer based on chromosomal loss and microsatellite instability predicts survival. Clin Cancer Res 8: 2311-2322, 2002.

46. Halling KC, French AJ, McDonnell SK, Burgart LJ, Schaid DJ, Peterson BJ, Moon-Tasson L, Mahoney MR, Sargent DJ, O'Connell MJ, et al: Microsatellite instability and 8p allelic imbalance in stage B2 and C colorectal cancers. J Natl Cancer Inst 91: 1295-1303, 1999.

47. Hemminki A, Mecklin JP, Järvinen H, Aaltonen LA and Joensuu H: Microsatellite instability is a favorable prognostic indicator in patients with colorectal cancer receiving chemotherapy. Gastroenterology 119: 921-928, 2000.

48. Samowitz WS, Curtin K, Ma KN, Schaffer D, Coleman LW, Leppert M and Slattery ML: Microsatellite instability in sporadic colon cancer is associated with an improved prognosis at the population level. Cancer Epidemiol Biomarkers Prev 10: 917-923, 2001

49. Watanabe T, Wu TT, Catalano PJ, Ueki T, Satriano R, Haller DG, Benson AB III and Hamilton SR: Molecular predictors of survival after adjuvant chemotherapy for colon cancer. N Engl J Med 344: 1196-1206, 2001

50. Salazar R, Roepman P, Capella G, Moreno V, Simon I, Dreezen C Lopez-Doriga A, Santos C, Marijnen C, Westerga J, et al: Gene expression signature to improve prognosis prediction of stage II and III colorectal cancer. J Clin Oncol 29: 17-24, 2011.

51. Gray RG, Quirke P, Handley K, Lopatin M, Magill L, Baehner FL, Beaumont C, Clark-Langone KM, Yoshizawa CN, Lee $\mathrm{M}$, et al: Validation study of a quantitative multigene reverse transcriptase-polymerase chain reaction assay for assessment of recurrence risk in patients with stage II colon cancer. J Clin Oncol 29: 4611-4619, 2011.

52. O'Connell MJ, Lavery I, Yothers G, Paik S, Clark-Langone KM, Lopatin M, Watson D, Baehner FL, Shak S, Baker J, et al: Relationship between tumor gene expression and recurrence in four independent studies of patients with stage II/III colon cancer treated with surgery alone or surgery plus adjuvant fluorouracil plus leucovorin. J Clin Oncol 28: 3937-3944, 2010. 
53. Deschoolmeester V, Baay M, Lardon F, Pauwels P and Peeters M: Immune cells in colorectal cancer: Prognostic relevance and role of MSI. Cancer Microenviron 4: 377-392, 2011.

54. Eveno C, Nemeth J, Soliman H, Praz F, de The H, Valleur P, Talbot IC and Pocard M: Association between a high number of isolated lymph nodes in T1 to T4 N0M0 colorectal cancer and the microsatellite instability phenotype. Arch Surg 145: 12-17, 2010.

55. Kuperwasser C, Chavarria T, Wu M, Magrane G, Gray JW, Carey L, Richardson A and Weinberg RA: Reconstruction of functionally normal and malignant human breast tissues in mice. Proc Natl Acad Sci USA 101: 4966-4971, 2004.

56. Kalluri R: Basement membranes: Structure, assembly and role in tumour angiogenesis. Nat Rev Cancer 3: 422-433, 2003.

57. Pavlides S, Whitaker-Menezes D, Castello-Cros R, Flomenberg N, Witkiewicz AK, Frank PG, Casimiro MC, Wang C, Fortina P, Addya S, et al: The reverse Warburg effect: Aerobic glycolysis in cancer associated fibroblasts and the tumor stroma. Cell Cycle 8: 3984-4001, 2009.
58. Bonuccelli G, Tsirigos A, Whitaker-Menezes D, Pavlides S, Pestell RG, Chiavarina B, Frank PG, Flomenberg N, Howell A, Martinez-Outschoorn UE, et al: Ketones and lactate 'fuel' tumor growth and metastasis: Evidence that epithelial cancer cells use oxidative mitochondrial metabolism. Cell Cycle 9: 3506-3514, 2010.

59. Martinez-Outschoorn UE, Balliet RM, Rivadeneira DB, Chiavarina B, Pavlides S, Wang C, Whitaker-Menezes D, Daumer KM, Lin Z, Witkiewicz AK, et al: Oxidative stress in cancer associated fibroblasts drives tumor-stroma co-evolution: A new paradigm for understanding tumor metabolism, the field effect and genomic instability in cancer cells. Cell Cycle 9: 3256-3276, 2010.

60. Worthley DL, Giraud AS and Wang TC: Stromal fibroblasts in digestive cancer. Cancer Microenviron 3: 117-125, 2010. 